\title{
Revizyon ve re-revizyon total diz protezi uygulamalarında karşılaşılan problemler
}

\section{Intraoperative difficulties in revision and re-revision total knee arthroplasty}

\author{
Şükrü Araç, Hakan Boya \\ Başkent Üniversitesi, Zübeyde Hanım Uygulama ve Araştırma Merkezi, Ortopedi ve Travmatoloji Departmanı, İzmir
}

Revizyon ve re-revizyon total diz artroplastileri kompleks cerrahi uygulamalardır. Bu ameliyatlar sırasında cerrahlar, üstesinden gelmeleri gereken birçok zorlukla karşılaşırlar. Başarılı sonuçlar elde edebilmek için, bu cerrahi uygulamanın tüm yönlerini ayrıntılarıyla anlamış olmak gerekir. Bu yazıda, bu tip olguların cerrahisinde karşılaşılan zorluklar mercek altına alınmıştır.

Anahtar sözcülkler: revizyon; re-revizyon; diz; artroplasti; komplikasyonlar; teknik zorluklar
Revision and re-revision total knee arthroplasty are complex procedures. There are many technical intraoperative difficulties that surgeons have to overcome. For satisfactory results, we should fully understand all aspects of the procedure in detail. This article focuses on the difficulties in such cases.

Key words: revision; re-revision; knee; arthroplasty; complications; technical difficulties

- Uzun ve longitudinal uzanan skarlar varsa, skar yeni insizyon içine alınmalı veya yeni insizyon eski skarın lateralinde, ona paralel olacak şekilde ve mümkün olduğu kadar uzakta (en az $7 \mathrm{~cm}$ ) yapılmalıdır.

- İki eski insizyon skarı varsa, yeni cilt insizyonu uzun olan üzerinden yapılmalıdır.

- Çok riskli olgularda "doku genişleticiler” (plastik cerrahi konsültasyonu) kullanılabilir.

Ekstansör mekanizma, yumuşak doku örtüsünün bir parçasıdır. ${ }^{[1]} \mathrm{Bu}$ olgularda ekstansör mekanizma fibrotiktir ve patellar tendon kalınlaşmıştır. Cerrahi uygulama sırasında diz yeterince fleksiyona getirilemez ve sonuçta ekstansör mekanizma hasarı (kuadriseps tendon rüptürü, patella kırığı, patellar tendon rüptürü) oluşabilir. Hem diz fleksiyonunu arttırmak hem de ekstansör mekanizma gerginliğini azaltmak için, artrotomi alternatifleri bilinmelidir.

Mediyal parapatellar artrotomi, bu olgularda genellikle yeterli açılım sağlamaz. Olguların bir kısmı için, mediyal parapatellar artrotomi ile beraber, ek uygulamalar yapılması gerekli olabilir; ${ }^{[4]}$

- Küçük skarlar varsa, yeni insizyon eski skarla geniş açı ile birleşmelidir.

- İletişim adresi: Prof. Dr. Şükrü Araç, Başkent Üniversitesi, Zübeyde Hanım Uygulama ve Araştırma Merkezi, Ortopedi ve Travmatoloji Departmanı, Caher Dudayev Bulvarı, No: 175, Bostanlı, Karşıyaka, İzmir Tel: 0532 - 2654276 e-posta: sukru.arac@deu.edu.tr

- Geliș tarihi: 8 Nisan 2015 Kabul tarihi: 8 Nisan 2015 


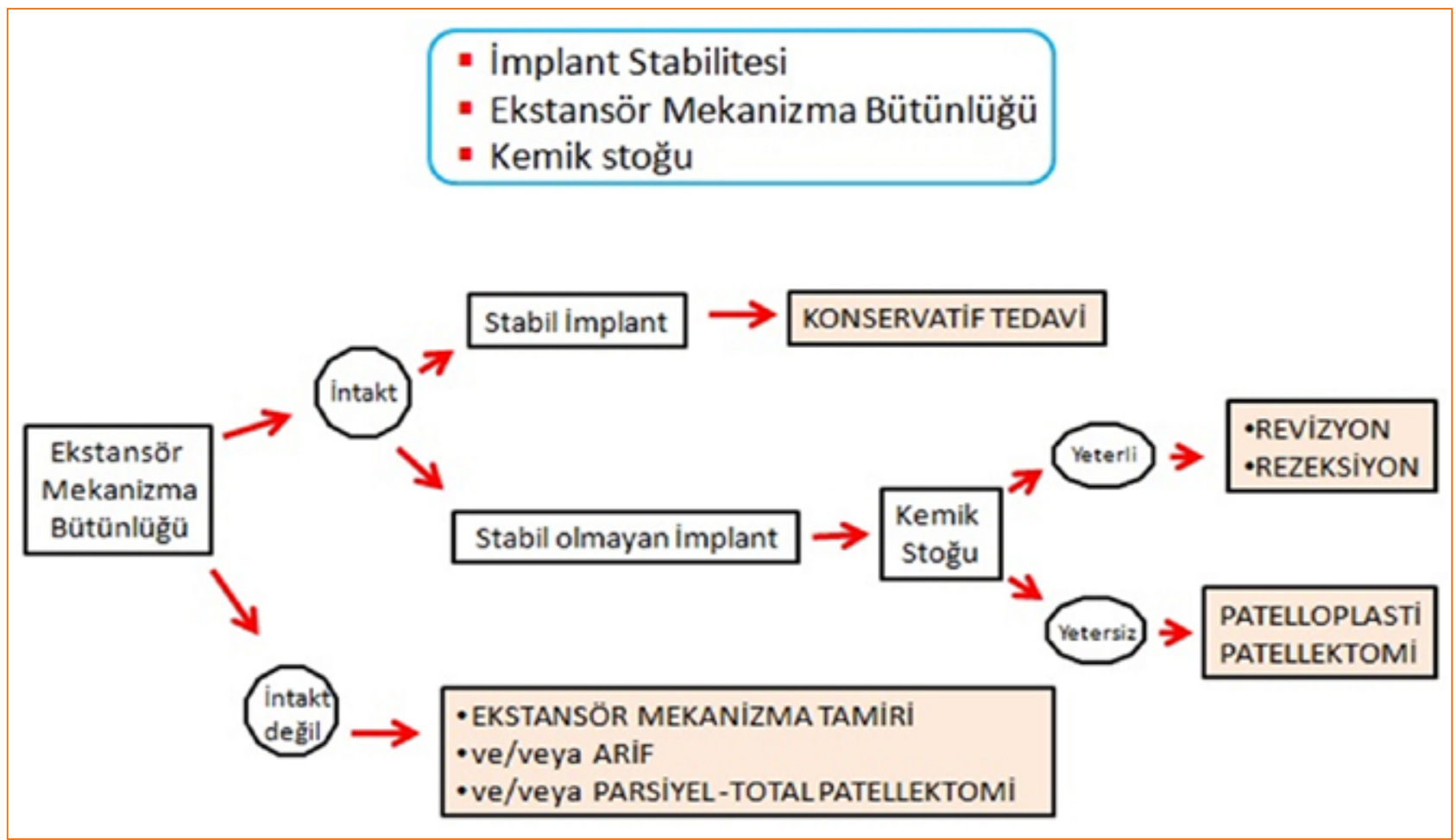

Şekil 1. Periprostatik patella kırıklarında tedavi planlaması.

- Kuadriseps tendonu (KT) ile distal femur arasındaki yapışıklıkların serbestleştirilmesi,

- Femur mediyal ve lateral kondilleri üzerindeki kapsül yapışıklıklarının serbestleştirilmesi,

- Epikondiller üzerinden distale doğru serbestleştirme (kollateral bağların yüzeyinde) ve

- Skarlı kapsül dokusunun inceltilmesi, lateral retinakuler gevşetme, patellar tendon (PT) skar dokusu eksizyonu mutlak olarak yapılmalıdır.

Bu ek uygulamaların da yetersiz kaldığı durumlarda;

- Kuadriseps kırpma,

- V-Y kuadrisepsplasti,

- kuadrisepsin tendonunun döndürülüp aşağı devrilmesi (turndown) ve

- Tibial tüberkül osteotomisi gibi ek yöntemler kullanılabilir; $[1,2,4-7]$

Nadir olarak kullanılsa da, cerrahi açılımın sağlanması için;

- Femoral soyma (peel),

- Epikondiler osteotomi,

- Tibial tüberkül osteomiyofasyal flep ve

- Tibial tüberkülün muz gibi soyulması gibi alternatif yöntemler de seçilebilir; ${ }^{[4,8,9]}$
Kuadriseps tendonun ameliyat sırasında rüptürü[10-12] çok nadirdir. Tedavideki hedef, transosseöz sütürler ya da sütür ankorlar kullanılarak, primer tamir yapmaktır. Primer tamir, tek başına yeterli değildir ve mutlak olarak Scuderi turndown tekniği, Dacron bant veya allogreft ile desteklenmesi önerilmektedir.

$\mathrm{Bu}$ olgularda, ameliyat sırasında patellar tendonun rüptürü[10-12] kuadriseps tendonunun rüptürüne göre daha sık izlenir. Primer tamir yapılsa da yeterli olmaz. Serklaj teli, semitendinozus/grasilis/kuadriseps otogrefti, sentetik greftler/bağ veya allogreft kullanılarak primer tamirin desteklenmesi gerekir. Bu işlemler destekleyici uygulamalar olarak tanımlanmış olsa da, aynı zamanda rekonstrüksiyon yöntemleridir.

Ameliyat sırasında patella kırıkları ${ }^{[1,10-13]}$ ile karşılaşılabilir. Patella kırıklarının tedavisi planlanırken, implant stabilitesi, ekstansör mekanizma bütünlüğü ve kemik stoğu dikkatle değerlendirilmelidir (Şekil 1).

Patellar protezin revizyonunu[14-16] planlamak karmaşık bir konudur. Patellar komponentin olmadığı durumlarda, patellofemoral eklem semptomatik ise, patellar protez uygulaması düşünülebilir. Patellar komponentin mevcut olduğu olgularda ise; aşınma, gevşeme, enfeksiyon varlığı, yeni femoral komponentin trokleası ile olan uyumu değerlendirilerek revizyon kararı verilir. Reimplantasyon planlanan olgularda, mevcut komponent 


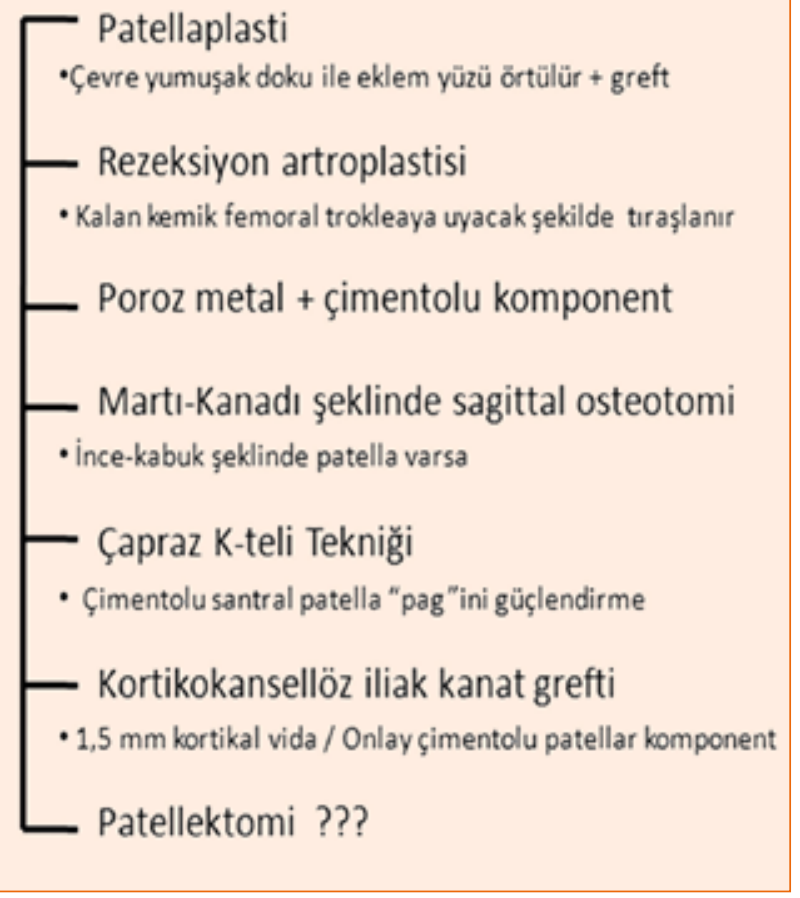

Şekil 2. Yetersiz patellar kemik stoğu varlığında, patellar protez revizyonu için alternatif uygulamalar.

çıkartıldıktan sonra patellanın kemik kalitesi ve kemik stoğunun durumu değerlendirilir. Yeni bir patellar protez uygulaması için, patella kalınlığı en az 10 mm olmalıdır; $15 \mathrm{~mm}$ altındaki patella kalınlığı varlığında kırık riskinin artacağını unutmamak gerekir. Kemik kalitesi ve stoğu yeterli ise re-implantasyon planlanır. Şayet kenarlar sağlamsa ve santral defekt varsa, içine uygulama (inlay) yöntemi kullanılabilir ve bikonveks patellar komponent tercih edilir. Aksi durumda, yüzey düzeltilir ve üzerine uygulama (onlay) yapılır. Kemik kalitesi ve stoğunun yetersiz olduğu durumlarda ise farklı uygulamalar tercih edilebilir (Şekil 2).

Patellektomisi ${ }^{[15,16]}$ olan hastalarda, patellanın biyomekanik avantajını tekrar elde edebilmek için, ekstansör mekanizmada patella lokalizasyonunda subsinoviyal poşa kemik grefti veya poroz metal yerleştirilebilir, ekstansör mekanizma tübülerize edilebilir ya da total ekstansör mekanizma allogrefti kullanılabilir.

Re-revizyon olgularında nadir görülse de, majör ekstansör mekanizma kaybi ${ }^{[17,18]}$ (KT+Patella+PT+kapsül+cilt defekti) önemli bir problemdir. Cilt ve kapsül defekti olduğu için, total ekstansör mekanizma allogreftleri kullanılamaz. Proksimalde de defekt olduğundan, gastroknemius kas-tendon flebi kullanımı yetersiz kalır. Serbest kas flepleri ise yalnızca yumuşak doku örtüsü sağlar, motor güce katkıda bulunmaz; ayrıca, uygulamak için mikrovasküler cerrahi gereklidir ve bunlar, donör alan morbiditesi oluşturur. Bu olgularda, vastus lateralis ve ya vastus mediyalis kas-tendonu, kapsül ve sinovyalarıla birlikte, tuberositas tibiaya transfer edilebilir (lokal kas transferi). Bu yöntem, bir taraftan yumuşak doku örtüsü oluştururken diğer taraftan, kısmen de olsa, kaudriseps kas gücünü restore edebilir.

Revizyon cerrahilerinde en sık peroneal sinir hasarı ${ }^{[1]}$ izlenir; direkt yaralanma çok nadirdir. Genelde ameliyat sırasında, traksiyon, hematom basısı veya uzamış turnike kullanımına bağlı olarak ortaya çıkar. Bu grup hastada, lomber sinir basılarının varlığının da (double-crush fenomeni) risk faktörü olduğunun bilinmesi gerekir.

Revizyon olgularında arter yaralanması[ ${ }^{[1,19]}$ sıklığı $\% 0,36$ olarak rapor edilmiştir. Diz arkasında, popliteal damarları örten sert skar dokusunun eksizyonu sırasında, damar hasarı oluşabilir. Diz fleksiyonu ile beraber popliteal arterin arkaya doğru yer değiştirdiğine inanılsa da, arterin \%15 sıklıkta proksimal tibia posterior yüzeyine, öne doğru hareket ettiği gösterilmiştir. Arter yaralanmaları; klinik olarak, iskemi, kanama, yalancı anevrizma veya kanama ve iskemi birlikteliği şeklinde karşımıza çıkabilir.

Şüphesiz ki eski implantın çıkartılması ${ }^{[20]}$ hem zordur hem de titizlikle planlanmalıdır. Bu işlem sırasında; kemik stoğu kaybı, ameliyat sırasında kırık veya bağ hasarı oluşabilir. Bu işlemin başarı ile yapılabilmesi için, yeterli cerrahi açılımın sağlanmış olması gerekir.

Revizyon olgularında, ameliyat sırasında periprostetik kırık ${ }^{[21]}$ sıklığı $\% 0,78$ olarak bildirilmiştir. Hastaların kemik stoğu kırılgan ve zayıf olduğu için, kırıklara sık rastlanır. Bu durum, özellikle implantların çıkartılması sırasına ortaya çıkar.

Stabil eklem için gerekli olan, uygun ekstremite dizilimi ile sağlam ve dengeli kollateral bağlardır. Instabilite ${ }^{[1,22-26]}$ tanısı ile yapılan revizyon sıklığı \%4,930 arasındayken, revizyon sonrası, olguların \%22'sinde instabilitenin farklı düzeylerde devam ettiği bildirilmiştir. Genel instabilite nedenlerinin net olarak bilinmesi gerekir (Tablo 1). Eğer komponentler uygunsuz yerleştirilmişse, instabilite sıklığı \%60-70'e ulaşabilir. Revizyon cerrahisinde instabilite ile baş edebilmek için, ameliyat öncesi dönemde fizik muayene ve uygun radyolojik değerlendirme (ortoröntgenografi, stres, yüklenme ön-arka ve lateral radyografileri) titizlikle yapılmalıdır. Üç tip instabilite vardır: fleksiyon, ekstansiyon ve rekurvatum. Özellikle $30-60^{\circ}$ fleksiyonda izlenen midfleksiyon instabilitesinin önüne geçmek için, eklem çizgisinin distale taşınarak normal anatominin oluşturulması ve femoral komponentin posterior offset'inin restorasyonu hayati önem taşır. 
Tablo 1. İnstabilite nedenleri

\begin{tabular}{ll}
\hline Erken $(<2$ yıl $)$ instabilite & Geç instabilite \\
\hline Ameliyat sırasında bağ hasarı & Polietilen aşınması \\
Komponent "malalignement"ı & Aseptik gevşeme/komponent migrasyonu \\
Koronal stabilizatörlerin aşırı gevşetilmesi & Geç bağ yetmezliği \\
& Küçük femoral komponent \\
& Aşırı tibial slop \\
& Aşırı eklem çizgisi elevasyonu
\end{tabular}

Tablo 2. Anderson Ortopedi Araştırma Enstitüsü’nün kemik defektleri sınıflaması

\begin{tabular}{ll}
\hline Tip & Kemik defekti boyutu \\
\hline 1 & Minör femoral veya tibial defekt, metafizyel kemik sağlam, revizyon komponent stabilitesi etkilenmez. \\
2 & Metafizyel kemik hasarı var, kansellöz femoral metafizyel kemik kaybı, revizyon komponent stabilitesi için rekonstrüksiyon gerektirir. \\
a) Defektler bir femoral veya bir tibial kondilde. & b) Defektler her iki femoral veya tibial kondilde. \\
3 & $\begin{array}{l}\text { Metafizyel segment defekti femoral kondil ya da tibia platosunun majör bölümünü etkiler, beraberinde bazen kollateral ligaman } \\
\text { veya patellar tendon ayrılması izlenebilir. }\end{array}$
\end{tabular}

Tablo 3. Revizyon total diz protezi uygulamalarında kemik kaybının tedavisi

\begin{tabular}{lll}
\hline Defekt tipi & & Tedavi seçeneği \\
\hline Kaviter defekt & $<5 \mathrm{~mm}$ & Kemik çimentosu ile doldurma \\
& $5-10 \mathrm{~mm}$ & Desteklenmiş kemik çimentosu \\
& $>10 \mathrm{~mm}$ & Morsalize allogreft veya poroz metal destekler \\
Segmenter defekt & $<5 \mathrm{~mm}$ & Kemik çimentosu \\
& $5-10 \mathrm{~mm}$ & Desteklenmiş kemik çimentosu \\
& $(<\% 50 \mathrm{femur}$ kondili / tibia platosu $)$ & \\
& $5-15 \mathrm{~mm}$ & Stem ve destek içeren modüler sistemler \\
& $(<\% 50 \mathrm{femur}$ kondili / tibia platosu $)$ & \\
& $>15 \mathrm{~mm}$ & Yapısal allogreft, megaprotez, poroz metal destek
\end{tabular}

Eklem çizgisinin restorasyonu ${ }^{[27,28]}$ önemli bir hedeftir. Re-revizyon olgularında, distal kemik kaybı dikkatle değerlendirilmelidir ve mutlaka, femoral komponentin anatomik eklem çizgisi elde edilinceye kadar distalizasyonu planlanmalıdır. Normal anatomiye kıyasla, diz ekstansiyonda iken eklem çizgisinin 4 mm'den fazla elevasyonu veya distalizasyonunun kötü sonuçlara neden olduğu bilinmektedir. Diz fleksiyon pozisyonundayken eklem çizgisi seviyesinin temel belirleyicisi, posterior femoral kondil yüksekliğidir.

Bu tür revizyon olgularında, kemik stoğu kaybı ${ }^{[29]}$ önemli bir problem olarak karşımıza çıkar. Kemik stoğu kaybına; mekanik gevşeme, enfeksiyon, osteolizis, implant ve çimentonun çıkarılması, antibiyotikli spacer neden olabilir. Tedavinin hedefi, hem aktüel revizyon sırasında stabiliteyi sağlamak hem de daha sonraki revizyonlar için kemik stoğu restorasyonudur. Tedavi sırasında önemli olan iki temel parametre, defektin boyutu ve lokalizasyonudur. Klinikte kemik defektlerinin tanımlanması için sıklıkla "Anderson Ortopedi Enstitüsü" sınıflaması kullanılır (Tablo 2). Kaviter ya da segmenter defektler için, defektin boyutu ve lokalizasyonuna göre farklı tedavi yöntemleri kullanılabilir (Tablo 3).

Protez seçimi ${ }^{[30]}$ önemli bir problemdir ve cerrahın bu tip olgularda uygun değerlendirme yaparak doğru protez tipini belirlemesi gerekir. Bu olgulara farklı düzeylerde kemik stoğu kaybı ve yumuşak doku laksitesi eşlik eder. Mevcut duruma uygun olan en az kısıtlayıcı özelliği olan protezin kullanılması gerekir. Arka çapraz bağın korunduğu tasarımın minimal yapısal stabilitesi vardır ve sağlam kollateral bağlar, sağlam arka çapraz bağ ve yeterli düzeyde kemik stoğunun bulunduğu olgularda kullanılabilir. Bu tip protezlerin kullanımına 
uygun şartlar, revizyon olgularında genellikle bulunmaz. Arka çapraz bağın yerine geçen posterior stabilize (PS) tasarımlarda yeterli antero-posterior stabilite elde edilir, fakat bu tasarımın koronal plan stabilitesine ve rotasyonel stabiliteye herhangi bir katkısı bulunmaz. Fleksiyon boşluğu dengeli ve yeterince gergin, kollateral bağlar sağlam olmalı, yeterli kemik stoğu bulunmalıdır. Komponentler arasında bağlantının olmadığı, varus-valgus hareketlerini engelleyen tasarım, PS tasarımlara göre daha fazla varus-valgus ve rotasyonel stabilite sağlar. Mobil yüklenme yüzeyi olan, bağlantısız varus-valgus hareketlerini engelleyen tasarımlar ise, fiksasyon ortak yüzeyine aktarılan stresleri ve posterior stabilizasyon mekanizmasına etki eden rotasyonel stresleri azaltır. Illeri fleksiyon instabilitesi, kollateral bağ kaybı, masif kemik kaybı ve posterior kapsüler yetmezlikle beraber kontrolsüz hiperekstansiyon varlığında, menteşeli protezler kullanılabilir. Rotasyonel serbestliği olanlar, sabit olanlara tercih edilir.

\section{KAYNAKLAR}

1. Kempshall PJ, Sharma H, Morgan-Jones RL. Revision total knee arthroplasty: complications. J Orthop Traum 2012;26(2):95-111. CrossRef

2. Cho W. Knee Joint Arthroplasty. Berlin, Heidelberg: SpringerVerlag; 2014.

3. Clarke HD, Scuderi GR. Revision total knee arthroplasty: planning, management, controversies and surgical approaches. Instr Course Lect 2001;50:359-65.

4. Eng GA. Exposure options for revision total knee arthroplasty. In: Bono JV, Scott RD, editors. Revision Total Knee Arthroplasty. New York: Springer; 2005. p.63-73. CrossRef

5. Garvin LG. Surgical Exposures-Quadriceps Snip. In: Scuderi GR, Tria AJ Jr, editors. Techniques in Total Knee Arthroplasty. New York: Springer Verlag; 2002. p.149-54.

6. Scuderi GR. The utility of the $\mathrm{W}$ quadricepsplasty in revision total knee arthroplasty. Tech Knee Surg 2007;6(2):85-7.

7. Hocking RA, Bourne RB. Tibial tubercle osteotomy in revision total knee replacement. Tech Knee Surg 2007;6:88-92.

8. Huff TW, Windsor RE. Difficult exposures in total knee arthroplasty: the femoral peel. Curr Orthop Pract 2008;19(3):272-5.

9. Roehring GS, Kang M, Scuderi GR. Surgical exposure for the complex revision total knee arthroplasty. Tech Knee Surg 2009;8(3):154-60.

10. Brooks P. Extensor mechanism ruptures. Orthopaedics 2009;32(9):pii:orthosupersite.com/view.asp?rID=42852. CrossRef

11. Pagnano MW. Patellar tendon and quadriceps tendon tears after total knee arthroplasty. J Knee Surg 2013;16(4):242-7.
12. Deirmangian CA, Lonner JH. Extensor Mechanism Rupture. In: Brown TE, Cui Q, Mihalko WM, Saleh KJ, editors. Arthritis \& Arthroplasty: Knee. Philadelphia, PA: Saunders Elsevier; 2009. p.288-95.

13. Dalury DF, Dennis DA. Extensormechanism problems following total knee replacement. J Knee Surg 2003;16(2):118-22.

14. Maheshwari AV, Tsailas PG, Ranawat AS, Ranawat CS. How to address the patella in revision total knee arthroplasty. Knee 2009;16(2):92-7. CrossRef

15. Dennis DA, Berend ME. Extensor Mechanism Complications. In: Berry DJ, Trousdale RT, Dennis DA, Paprosky WG, editors. Revision Total Hip and Knee Arthroplasty. Philadelphia, PA: Lippincott, Williams \& Wilkins; 2012. p.559-69.

16. Deirmengian GK, Israelite CL. Patella Revision/ Reconstruction. In: Brown TE, Cui Q, Mihalko WM, Saleh $\mathrm{KJ}$, editors. Arthritis \& Arthroplasty: Knee. Philadelphia, PA: Saunders Elsevier; 2009. p.247-56.

17. Whiteside LA. Surgical technique: vastus medialis and lateralis as flep transfer for knee extensor mechanism deficiency. Clin Orthop Relat Res 2013;471(1):221-30. CrossRef

18. Whiteside LA. Surgical technique: Muscle transfer restores extensor function after failed patella-patellar tendon allogreft. Clin Orthop Relat Res 2014;472(1):218-26. CrossRef

19. Shetty AA, Tindall AJ, Qureshi F, Divekar M, Fernando KW. The effect of knee flexion on the popliteal artery and its surgical significance. J Bone Joint Surg Br 2003;85(2):218-22.

20. MasonJB, Fehring TK. Removing well-fixed total knee arthroplasty components. Clin Orthop Relat Res 2006;446:76-82.

21. Vince KG. Modes of failure in total knee arthroplasty. In: Advanced reconstruction knee. 978-0-89203-452-9 AAOS; 2011. p.3141-55.

22. Pulido L, Parvizi J, Macgibeny M, Sharkey PF, Purtill JJ, Rothman RH, Hozack WJ. In hospital complications after total joint arthroplasty. J Arthroplasty 2008;23(6 Suppl 1):139-45. CrossRef

23. Azzam K, Parvizi J, Kaufman D, Purtill JJ, Sharkey PF. Austin MS. Revision of the unstable total knee arthroplasty outcome predictors. J Arthroplasty 2011;26(8):1139-44. CrossRef

24. Del Gazio DJ, Della Valle CJ. Instability in primary total knee arthroplasty. Orthopedics 2011;34(9):e519-21. CrossRef

25. Parrate $S$, Pagnanao MW. Instability after total knee arthroplasty. J Bone Joint Surg Am 2008;90(1):184-94.

26. Clarke HD, Scuderi GR. Flexion instability in primary total knee replacement. J Knee Surg 2003;16(2):123-8.

27. Hofmann AA, Kurtin SM, Lyons S, Tanner AM, Bolognesi MP. Clinical and radiographic analysis of accurate restoration of the joint line in revision total knee arthroplasty. J Arthroplasty 2006;21(8):1154-62.

28. Bellemans J. Restoring the joint line in revision TKA: does it matter? The Knee 2004;11(1):3-5.

29. Lombardi AV, Berend KR, Adams JB. Management of bone loss in revision TKA: it's a changing world. Orthopedics 2010;33(9):662. CrossRef

30. Johnson DR, Dennis DA, Kim RH. How much constraint is required in complex revision total knee arthroplasty. Tech Knee Surg 2009;8(3):166-73. 